\title{
Considering new insights into antisociality and psychopathy
}

ARTICLE in THE LANCET PSYCHIATRY · FEBRUARY 2015

DOI: 10.1016/S2215-0366(14)00125-4

CITATIONS

2

1 AUTHOR:

Inti A. Brazil

Radboud University Nijmegen

23 PUBLICATIONS 155 CITATIONS

SEE PROFILE

\section{READS}

81 


\section{Considering new insights into antisociality and psychopathy}

Sarah Gregory and colleagues ${ }^{1}$ report a functional MRI study of violent offenders with antisocial personality disorder. 12 men with antisocial personality disorder with psychopathy, 20 men with antisocial personality disorder but not psychopathy, and 18 healthy non-offenders were assessed with an event-related probabilistic responsereversal task adapted for the scanning environment. Gregory and colleagues suggest that processing of punishment during response reversal could be a cognitive operation that distinguishes between people who have antisocial personality disorder with and without psychopathy. More specifically, they found increased activation in the posterior cingulate cortex and anterior insula in response to punished errors during the task reversal phase and decreased activation in the superior temporal cortex in response to all correct rewarded responses in offenders with antisocial personality disorder and psychopathy. These patterns were not seen in offenders without psychopathy. Although the focus of the study was on dissecting the neural properties of response reversal in these two subgroups, the results additionally speak to more general theoretical topics highly relevant for psychopathy research.

The findings emphasise the importance of continually reassessing the main arguments that fuel longstanding debates on the nature and conceptualisation of psychopathy and its relation to antisociality. Although it is easy to discard findings that provide evidence against whichever theoretical stance a scholar believes in most, it might be much more beneficial to reconsider preferences in the light of new data and develop new frameworks that integrate seemingly contradicting positions. The prevailing approach to diagnosing psychopathy in offender populations is a categorical one, in which those who score higher than the cutoff value on the psychopathy checklist are classified as being psychopathic. ${ }^{2}$ In the past two decades, however, competing frameworks have emerged that point to other causal pathways for psychopathy and antisociality through dimensional operationalisations. ${ }^{3.4}$ These different approaches have led to many new insights, but also to the polarisation of scholars. The results obtained by Gregory and colleagues, ${ }^{1}$ my laboratory, ${ }^{5}$ and many others with a categorical approach stress that there is still much that cannot be readily explained by taking a purely dimensional approach when studying clinical manifestations of psychopathy and See Articles page 153 antisocial personality disorder in adult offenders.

Importantly, the categorical and the dimensional approaches both have their merits and are supported by empirical research. Perhaps, therefore, both are correct. Many studies have looked at the unique explanatory power of the dimensions believed to underlie psychopathy and generic antisociality. Nevertheless, the often-neglected interactions between dimensional traits might be crucial to facilitating the emergence of unique personality, neurocognitive, and behavioural characteristics. A study on emotion interference on cognitive processing in a non-clinical sample has provided preliminary evidence for how the presence of specific interactions between psychopathy-related traits relate to three distinct cognitive-processing styles. ${ }^{6}$ Thus, in extreme populations, dimensional interactions could be linked to differences in neurocognitive and personality characteristics that are so fundamental that a category with its own dimensional properties might emerge at a socalled point of discontinuity. The notion that interacting personality dimensions are related to different cognitive and neurocognitive profiles within a category also fits well with empirical findings on the differential effects of high and low anxiety in psychopathy. ${ }^{8}$ Similarly, Gregory and colleagues' results support the idea that psychopathic traits interact with antisocial personality disorder, ${ }^{1}$ which creates a dichotomy that can also be seen in the distinct neurocognitive signatures between the groups.

Another interesting topic is that of the relation between abnormal brain activation and behavioural adaptation in psychiatric populations. Many therapeutic interventions aim to help patients modify or unlearn their maladaptive behavioural tendencies, which can lead to unfavourable outcomes. Sensitivity to outcome information signalling the need to adapt dominant responses is required. Gregory and colleagues ${ }^{1}$ report that offenders with antisocial personality disorder and psychopathy have increased activation to punished reversal errors relative to rewarded correct responses, whereas the pattern is opposite in those without psychopathy. Of note, despite this finding, these two groups did not differ on the behavioural level, perhaps because of the task parameters. ${ }^{9}$ Nevertheless, if impaired response reversal is a core feature of psychopathy, it could be argued that it should always be observable in behaviour 
without being affected by contextual factors, such as task parameters. ${ }^{10}$ The intact behavioural performance combined with aberrant brain activation reported, however, might reflect the differing developmental pathways for antisocial personality disorder with and without psychopathy. The distinction between these two subgroups resembles that between troubled youths with high levels of callous-unemotional traits and those predominantly with conduct disorder, pinpointing the presence of developmental components. Importantly, neural plasticity is high in the developing brain and, therefore, genetic, epigenetic, and environmental factors can shift brain organisation towards a different homeostatic balance in children with callous-unemotional traits compared with those without these traits to manage behaviour in daily life. From this perspective, the different patterns of activations observed in the violent offenders with antisocial personality disorder with and without psychopathy and healthy individuals might reflect these diverging neurodevelopmental pathways to cope with events signalling the need to adapt.

In sum, Gregory and colleagues provide intriguing new insights. Additionally, these lend themselves to reflecting about the complexity of antisocial personality disorder and psychopathy and, even more complex, their relations with neurocognitive functioning and behaviour.

\section{IA Brazil}

Donders Institute for Brain, Cognition and Behaviour, Radboud University, Nijmegen, Netherlands. Pompestichting, Nijmegen, Netherlands

i.brazil@donders.ru.nl

1 Gregory S, Blair RJ, Ffytche DH, et al. Punishment and psychopathy: a case-control functional MRI investigation of reinforcement learning in violent antisocial personality disordered men. Lancet Psychiatry 2014; 2: $153-60$.

2 Hare RD, Hart SD, Harpur TJ. Psychopathy and the DSM-IV criteria for antisocial personality disorder. J Abnorm Psychol 1991; 100: 391-98.

3 Lilienfeld SO, Andrews BP. Development and preliminary validation of a self-report measure of psychopathic personality traits in noncriminal populations. J Pers Assess 1996; 66: 488-524

4 Patrick CJ, Fowles DC, Krueger RF. Triarchic conceptualization of psychopathy: Developmental origins of disinhibition, boldness, and meanness. Dev Psychopathol 2009; 21: 913-38.

5 Brazil IA, Verkes RJ, Brouns BHJ, Buitelaar JK, Bulten BH, de Bruijn ERA. Differentiating psychopathy from general antisociality using the $\mathrm{p} 3$ as a psychophysiological correlate of attentional allocation. PloS one 2012; 7: e50339.

6 Maes JHR, Brazil IA. Distraction from cognitive processing by emotional pictures:preliminary evidence for an association with interactions between psychopathy-related traits in a non-clinical sample. Pers Indiv Diff 2015; 75: 53-58.

7 Putnam SP. Stability and instability of childhood traits: implications for personality development of animals. Dev Psychobiol 2011; 53: 510-20.

8 Koenigs M, Baskin-Sommers A, Zeier J, Newman JP. Investigating the neural correlates of psychopathy: a critical review. Mol Psychiatry 2011; 16: 792-99.

9 Finger EC, Marsh AA, Mitchell DG, et al. Abnormal ventromedial prefrontal cortex function in children with psychopathic traits during reversal learning. Arch Gen Psychiatry 2008; 65: 586-94.

10 Brazil IA, Maes JHR, Scheper I, et al. Reversal deficits in individuals with psychopathy in explicit but not implicit learning conditions. J Psychiatry Neurosci 2013; 38: E13-E20.

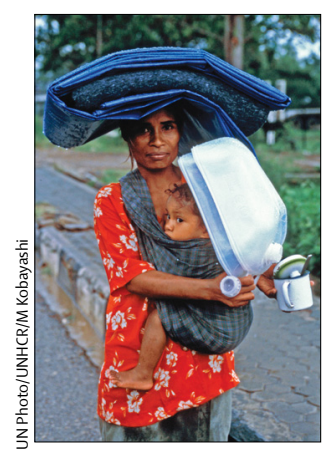

See Articles page 161

\section{Protecting the mental health of women in the perinatal period}

The modelling study reported in The Lancet Psychiatry by Derrick Silove and colleagues ${ }^{1}$ on perinatal depressive symptoms after mass conflict is based on years of work in post-conflict Timor-Leste, one of the poorest countries in Asia. The results, if confirmed, are relevant to the understanding and amelioration of perinatal mental health problems in vulnerable women. The study has been published just as attention is focusing on the need to include both the health of women and children ${ }^{2}$ and mental health ${ }^{3}$ in the new Sustainable Development Goals. Protection of women's mental health in the perinatal period is essential to this development agenda.

Perinatal mental disorders are highly prevalent worldwide, and depression is the most common and best recognised of these. ${ }^{4}$ These disorders cause suffering and economic and social problems for women and their families and communities. The growing recognition of the salience of these problems for countries of all types is described in a Lancet Series on this topic. ${ }^{4}$ Depression and other mental disorders in the perinatal period are common in low-income and middle-income countries (LMICs), despite the widespread view held until recently that these disorders were confined mainly to well resourced countries. ${ }^{4,5}$ These disorders are associated with increased risk of adverse health and development outcomes for children, especially in socioeconomically disadvantaged populations and low-income countries. In some LMICs, for instance, infant stunting and underweight are associated with depression in mothers. However, these adverse outcomes are not inevitable. ${ }^{4}$

A report from the London School of Economics in the UK describes the economic and social effects of maternal mental health problems in the perinatal period, defined as during pregnancy and the first year after childbirth. 